Ann. Biol. anim. Bioch. Biophys., I964. 4 (3), 2II-228.

\title{
ANALYSE STATISTIQUE DE LA SENSIBILITÉ A L'OCYTOCINE DE L'UTÉRUS DE RATTE IN VITRO
}

\author{
J. MARTINET et M. GILLOIS \\ Laboratoire de Physiologie de la Lactation \\ Station centrale de Génétique animale \\ Centre national de Recherches zootechniques, Jouy-en-Josas (Seine-et-Oise)
}

SOMMAIRE

La sensibilité de l'utérus de Ratte à l'ocytocine est testée en fonction du stade du cycle ovarien au cours duquel l'utérus est prélevé.

Cinq milieux de survie différents sont utilisés pour maintenir l'organe en survie (Pharmacopée britannique, 1953; Beauvillain, Ginsburg et Smith, Fitzpatrick, Holton).

$\mathrm{La}$ pente des droites de régression de la réponse utérine en fonction de la dose d'ocytocine appliquée est la meilleure pour les utérus choisis en prœstrus et en ostrus, maintenus en survie dans le milieu de la Pharmacopée britannique 1953 .

L'étude de l'extraction et de la purification de l'ocytocine sanguine nous a conduit à examiner les différentes techniques de dosage biologique de cette hormone, afin d'en définir les modalités et les limites d'utilisation. tocine :

Ces techniques biologiques font appel aux propriétés pharmacologiques de l'ocy-

- pouvoir ocytocique caractérisé par son activité sur la musculature utérine,

- pouvoir d'éjection par action sur le myoépithélium mammaire,

- activité dépressive vasculaire,

- activité sur les phénomènes de filtrations de certains ions à travers une membrane (rein ou vessie).

Nous nous arrêterons particulièrement sur la première de ces activités : la mesure du pouvoir ocytocique étant la propriété la plus couramment utilisée pour le dosage de l'ocytocine.

Dès le début du siècle, l'utilisation des contractions de l'utérus in vitro fut proposée comme moyen d'étalonner l'activité des extraits post-hypophysaires. L'es- 
pèce retenue fut d'abord le Cobaye (DALE et LAIDLAW, I9I2), puis le Rat (GuGGENHEIM, I9I4), qui fut ensuite admis officiellement comme espèce propre au dosage, car sa sensibilité, la fidélité et le pouvoir discriminatoire de ses réponses semblaient supérieurs à ceux du Cobaye (KNAUs et CLARK, I925).

Dans notre cas, le but étant le dosage de l'ocytocine sanguine, le pouvoir ocytocique étant toujours très faible, le Rat est l'espèce à laquelle nous ferons appel de préférence au cours de cette étude.

Au problème du choix de l'espèce s'ajoutent de nombreux facteurs qui retiendront notre attention : choix de l'utérus à un certain stade dans le cycle ovarien, composition du milieu de survie, température du bain, technique du dosage (contractions isométriques ou isotoniques - survie par perfusion ou en cuve).

\section{A. - Choix DU STADE DANS LE CYClE OVARIEN}

Le problème du choix de l'utérus selon le stade auquel se trouve l'ovaire, c'est-àdire selon les niveaux de motricité de l'utérus au cours du cycle, a fait l'objet de nombreux travaux. Certains auteurs étudient cette motricité en se basant sur l'activité spontanée et ses modifications par les extraits posthypophysaires, d'autres sur les contractions provoquées par ces extraits; les conditions techniques dtı dosage éliminant dans ce cas les contractions spontanées.

\section{I. - Activité spontanée de l'utérus au cours du cycle et ses modifications par les extraits posthypophysaires}

Dès I925, différents travaux soulignaient la relation probable entre le stade ovarien et l'activité utérine; seule la fréquence des contractions augmente lors de l'œstrus (DURRant et RosENFEL, I93I) ; le tonus et l'amplitude des contractions spontanées sont plus forts en proestrus et en œstrus (Clark, KNAUs et Parkes, I925; Brouha et Simonnet, I927) et plus faibles en dicestrus (HaRne, I932).

Cette activité spontanée de l'utérus est augmentée par l'addition de pituitrine à la solution de survie : la modification est plus importante en prœstrus et en œstrus (KNAUS et Clark, I925; HARNe, I932; SchneIDER et STUMPF, I953).

Les résultats sont identiques pour un utérus dont l'activité spontanée est enregistrée in vivo (GUERNE et STUTINSKY, Ig6r).

HARNE attribue ces modifications de la réactivité spontanée à la présence d'une substance liée au cycle ; elle serait en concentration maximum pendant le prostrus, puis diminuerait progressivement jusqu'au cycle suivant et ainsi de suite. Il est possible de déclencher artificiellement une activité utérine spontanée du type prœstrus ou œstrus chez des femelles impubères en leur injectant du liquide folliculaire (Brouha et Simonnet, I926, sur le Cobaye), chez des femelles castrées et traitées par les œstrogènes (GENELI, I940; RoBSON et SHARAF, I95I), l'activité ainsi déclenchée est augmentée par l'addition d'extraits posthypophysaires (DUFFNER et WHITEHEAD, I94I). 
Cette activité spontanée est particulièrement sensible à la température : elle croît en fréquence et en amplitude, lorsque la température du bain passe de Io à $3^{\circ}$ (Siaulis et Sollman, I927; Kumagar, Ebashi, Takeda, I952).

L'augmentation maximum de l'activité spontanée due à la pituitrine se produit vers $23^{\circ}$; cet accroissement d'activité devient de plus en plus faible, lorsque la température de la solution passe de 23 à $38^{\circ}$; toutefois, les auteurs ne précisent pas l'état physiologique de l'utérus étudié (KUMAGar, EBaShi, TAKEDA, I952).

\section{II. - Activité utérine déclenchée par l'addition d'ocytocine, variation en fonction du cycle ovarien}

En se plaçant dans des conditions de température (entre 25 et $3^{\circ}$ ) et de milieu convenablement choisies, il est possible d'éliminer toute activité spontanée et de déclencher ainsi les contractions par l'addition d'ocytocine au milieu de survie.

Selon les auteurs, les résultats sont assez contradictoires, pour certains l'amplitude des contractions est utilisable pour le dosage de l'ocytocine lorsque l'utérus est en prostrus (FLATTERS, I954) ; pour d'autres, en diostrus (BONNEY et FERGUSON, I950; KUMAGaI, EBAShI, TAKEDA, I952; Johnston, I958).

Enfin, une sensibilité satisfaisante serait obtenue en utilisant l'utérus de Rattes traitées un jour aux œstrogènes (FITZPATRICK, I96r) ou deux jours (WALKER, I96I) avant abattage pour le dosage.

\section{III. - Activité électrique de l'utérus de Ratte en fonction de son état physiologique}

Les techniques d'électromyographie ont tenté d'analyser les conditions de la motricité utérine. Elles ont confirmé la plus grande sensibilité de l'utérus de Ratte en prœstrus, en œstrus ou traités aux œstrogènes : sensibilité élevée aux stimulations électriques (MarshalL, I959; Kuriyama et Csapo, I959; Goto et Csapo, I959), augmentation du potentiel de membrane (MarshaLL, I959), trains de potentiels d'actions réguliers et liés aux contractions spontanées (BERGER et MARSHALI, I96I ; Marshal, I, Ig62).

Ces travaux ainsi que certains résultats contradictoires quant à l'importance du stade ovarien pour le choix des utérus à prélever en vue du dosage de microquantités d'ocytocine nous a conduits à étalonner l'aptitude de l'utérus à se contracter sous l'action de l'ocytocine en relation avec le cycle de l'ovaire ou après un prétraitement par injection d'œstrogène.

\section{B. - COMPOSITION DES SOLUTIONS DE SURVIE}

Les méthodes de dosage de l'ocytocine par mesure des contractions utérines in vitro sont caractérisées par la variabilité des milieux de survie utilisés. On peut se demander dans quelle mesure la composition de ces solutions influe sur la sensibilité de l'utérus et même sur la régression des réponses en fonction des substances ocytociques. 


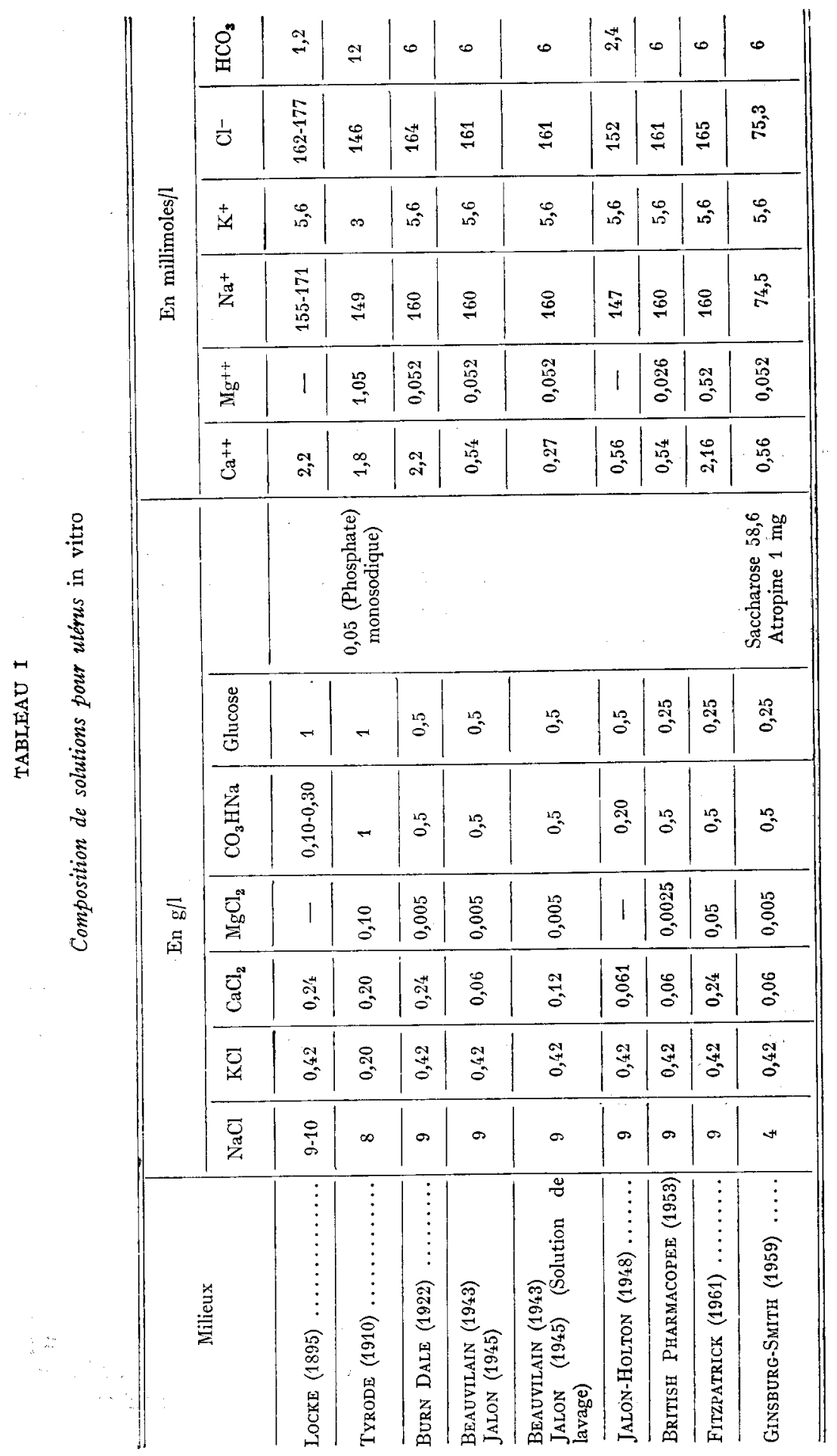


Les auteurs qui ont tenté d'interpréter l'effet des différents ions sur l'activité du muscle utérin se sont longuement arrêtés sur le rôle du calcium ; l'absence de $\mathrm{Ca}$ dans la solution suspend l'activité spontanée; l'activité électromyographique est faible (BERGER et MARShalL, I96I), la sensibilité à l'ocytocine est très réduite. Lorsque l'on augmente progressivement la concentration en calcium de o à $0,53 \mathrm{mM}$ de Ca par litre, on voit augmenter la sensibilité aux stimulations électriques (CsAPO, I956), l'activité électromyographique se développe (CSAPo et Kuriyana, I963), la sensibilité aux extraits posthypophysaires croît notablement (VAN DYKE, Ig28). $\mathrm{Au}$ delà de I mM de $\mathrm{Ca}$ les contractions spontanées se multiplient (VAN DyKE et HASTINGS, Ig28) les activités électriques du muscle utérin deviennent atypiques (Csapo et Kuriyama, Ig63).

D'autres travaux faisant varier la concentration des ions magnésium indiquent un optimum pour lequel la sensibilité à l'ocytocine est la plus grande ; ces résultats sont toutefois très variables; pour le Cobaye, cet optimum se place selon les auteurs à $0,05 \mathrm{mM}$ (BURN et DALE, I922) ou I mM par litre (VAN Dyke et HASTING, I928); pour le Rat, il se situerait vers $0,05 \mathrm{mM}$ par litre (GENELI, I938).

Les résultats sont plus homogènes en ce qui concerne le rôle du potassium extracellulaire dans la contraction musculaire utérine, avec toutefois des différences entre espèces : optimum entre 6,2 et 9,3 mM chez le Cobaye (VAN Dyke et Hasting, I928 ; KNAUS et CLARK, I925) à $5 \mathrm{mM}$ chez le Rat (JUNG, I96I).

Enfin, l'isotonicité étant maintenue par des ions inactifs ou par du saccharose, des essais ont été faits avec des milieux appauvris en sodium. Une réduction d'environ $50 \mathrm{p}$. 100 de sa concentration provoque une augmentation des potentiels de membrane (Csapo et KuRIYAMA, I963) et de fortes contractions spontanées suivies, après un délai d'une quinzaine de minutes, d'un retour aux conditions normales de sensibilité à l'ocytocine (MARShaII, I963).

Dans ce travail, nous avons repris un certain nombre de solutions de survie utilisées classiquement en pharmacologie, différentes par leur composition ionique, afin de juger de leur aptitude à développer des contractions utérines en présence de faibles doses d'ocytocine.

\section{MATÉRIEL ET MÉTHODF}

\section{A. - Milieux de survie utilisés}

Compte tenu des données bibliographiques, nous comparerons dans notre plan expérimental les solutions suivantes (tabl. I) :

\section{Io Solution de Beauvillain (1943).}

La composition de cette solution est comparable à celle de BuRn et DaLE (I922) (tabl. I) exception faite du chlorure de calcium qui y figure à un taux quatre fois plus faible.

$2^{\circ}$ Solution de la Pharmacopée britannique (1953).

Elle est caractérisée par un fajble taux de calcium comme la précédente, mais avec deux fois moins de magnésium.

$3^{0}$ Solution de Jalon-Holton décrite par Holton (1948).

Cette solution n'est" pas celle de JaLON telle qu'elle est publiée par l'auteur. Le milieu original de Jalon (1945) est identique à celui de Bealvillain. Pendant les périodes de repos, l'auteur 
l'appauvrit à $20 \mathrm{p}$. Ioo de son taux de calcium et rétablit la concentration initiale en calcium au moment de l'application de la substance ocytocique.

HOLTON décrit la solution de JALON comme du LOCKE ( 1896$)$ avec $\mathrm{r} / 2$ de calcium et $\mathrm{r} / 2 \mathrm{de}$ glucose, nous l'avons composé conformément à cette description, c'est-à-dire sans magnésium. De ce fait, cette solution que nous appellerons JALON-HOLTON ressemble à celle de MUNSICK (I960). Elle nous permettra de juger de la sensibilité utérine aux faibles doses d'ocytocine, en absence de magnésium et avec un taux de calcium identique à la solution de BEAUvillain ou de la Pharma. copée britannique.

\section{$4^{\circ}$ Solution de Ginsburg et Smith (I959).}

Le milieu de Ginsburg et Smith (1959) utilisé pour les dosages de l'ocytocine sur l'utérus de Rat maintenu en survie par la technique de perfusion (GADDUM, 1953) permet le dosage de quantité très faible d'ocytocine.

Il est caractérisé par un taux de calcium et de magnésium identique à la solution de BEAUVILIAIN. Il se singularise par une concentration en sodium environ deux fois plus faible que dans les milieux classiques. L'isotonicité est maintenue par l'addition de saccharose.

$5^{\circ}$ La solution de FrTZPatRick (1961), très riche en calcium et en magnésium, est utilisée par l'auteur avec la technique de perfusion pour le dosage de faible quantité d'ocytocine.

Ces différents milieux sont préparés à partir de solutions mères ne contenant ni glucose, ni saccharose, ni bicarbonate de soude. La solution définitive est préparée extemporanément.

\section{B. - Choix des utérus}

Les utérus de Rattes proviennent d'animaux de race Wistar (souche JouY) pesant I 30 à I $90 \mathrm{~g}$. Les frottis vaginaux (LONG et EvaNs, I922) sont suivis quotidiennement pendant deux semaines avant l'utilisation de l'animal. Celui-ci est donc abattu au stade du cycle ovarien correspondant exactement aux besoins du plan expérimental. Les 4 stades du cycle retenus pour cette expérience sont : prœstrus, œstrus, métœestrus et diœstrus. Les animaux à frottis douteux ne sont pas utilisés.

L'abattage de l'animal se fait par décapitation. L'utérus est disséqué et prélevé immédiatement. La corne utérine est sectionnée à quelques millimètres de chacune de ses extrémités et mise immédiatement dans un milieu de survie avant d'être montée dans la cuve à organe isolé.

$$
\text { C. - Matériel }
$$

La cuve à organe isolé est de faible volume utile $(3-5 \mathrm{cc})$. Elle est maintenue à température constante par une circulation d'eau à $32^{\circ} \mathrm{C}$ dans une double enveloppe ; le milieu est équilibré par un mélange gazeux $\mathrm{CO}_{2} 5 \mathrm{p}$. Iо0, $\mathrm{O}_{2} 95 \mathrm{p}$. Ioo ; l'extrémité mobile de la corne utérine est en relation avec un stylet enregistreur au moyen d'un fil ; la tension du fil est de $\mathrm{I}, 7 \mathrm{~g}$ et le coefficient d'amplification par le stylet est de i I. La contraction enregistrée est isotonique. Ces données sont main. tenues constantes au cours de toute l'expérience.

\section{D. - Plan expérimental}

$\mathbf{1}^{\text {0 }}$ Le plan expérimental portant sur des Rattes abattues à différents moments de leur cycle normal est celui du carré latin. Le schéma en est le suivant :

Stade ostrien $A$

\begin{tabular}{|c|c|c|c|c|c|}
\hline$N^{\circ}$ du Rat & \multicolumn{5}{|c|}{ Séquence d'application des 5 milieux } \\
\hline I & $a$ & $b$ & $c$ & $d$ & $e$ \\
\hline II & $d$ & $e$ & $b$ & $a$ & $c$ \\
\hline III & $b$ & $c$ & $a$ & $e$ & $d$ \\
\hline IV & $e$ & $a$ & $d$ & $c$ & $b$ \\
\hline v & $c$ & $d$ & $e$ & $b$ & $a$ \\
\hline
\end{tabular}


Les milieux utilisés sont désignés par les lettres $a b c d e$ pour chaque case du carré latin. L'utérus de chaque Rat est soumis dans chacun des milieux à trois doses d'ocytocine choisies de telle façon que la concentration finale dans la cuve soit de (I) $\mathrm{I}_{1} \mathrm{IO}^{-4},(2) 2 \cdot \mathrm{IO}^{-4}$, (3) 3. $\mathrm{TO}^{-4}$ U.I./ml. Ces trois doses sont appliquées deux fois dans l'ordre suivant (I) (2) (3) (3) (2) (I). que :

Si nous prenons par exemple la première case en haut à gauche du carré larin, nous dirons

- le rat I abattu au stade A a été soumis dans le milieu $a$ aux doses I.2.3.3.2.I. d'ocytocine, puis dans le milieu $b$ aux doses $1.2 \cdot 3$, etc.

Les doses d'ocytocine sont ajoutées à un volume donné de liquide introduit dans la cuve, afin d'obtenir avec précision la dilution finale désirée.

La contraction utérine se produit I 5 à 60 secondes après cette addition, l'utérus est ensuite lavé deux fois avec la solution en usage, puis soumis à la dose d'ocytocine suivante; l'écart entre deux applications est de 3 minutes. Lors du passage d'un milieu à un autre, l'utérus est lavé 4 fois avant la première addition d'ocytocine.

$2^{\circ} \mathrm{Au}$ cours d'une deuxième série expérimentale, nous avons repris les milieux pour lesquels la régression dose-réponse était significative lors de la I re expérience et nous les avons appliqués à des utérus de Rattes intactes traitées par une injection de stilbœstrol :

$$
\begin{aligned}
& \text { soit } 5 \mu \mathrm{g} \text { par } 200 \mathrm{~g} \text { de poids vif } \\
& \text { soit } \text { ro } \mu \mathrm{g} \text { par } 200 \mathrm{~g} \text { de poids vif }
\end{aligned}
$$

I7 7 ì 18 heures avant leur abattage (FITZPATRICK, I96i).

\section{E. - Analyse statistique}

L'analyse de la variance a été effectuée séparément pour les données obtenues pour chacun des stades du cycle.

La droite de régression a été calculée pour deux éventualités :

a) réponse utérine - dose d'ocytocine

b) réponse utérine - - log dose d'ocytocine

Dans les deux cas, la linéarité a été testée. 1952).

Enfin, le classement des droites de régression a été fait par comparaison deux à deux (FINNEY,

\section{RÉSULTATS}

\section{I. - Analyse de la variance des réponses utérines en fonction du cycle ovarien}

L'analyse de la variance des réponses des utérus choisis aux différents stades du cycle ovarien figure au tableau 2.

Cette analyse révèle une importante variation attribuée aux animaux et cela à tous les stades. C'est toutefois la variation due aux milieux de survie qui est la plus importante.

La variation imputable à l'application de doses croissantes d'ocytocine est toujours hautement significative, sauf pour le diøstrus où elle n'est significative qu'au seuil de $5 \mathrm{p}$. roo.

Le fait de répéter deux fois la même dose n'entraîne pas de variation de la réponse.

Enfin, la source de variation représentée par la place variable d'un milieu donné dans la succession séquentielle des solutions n'est jamais significative. 


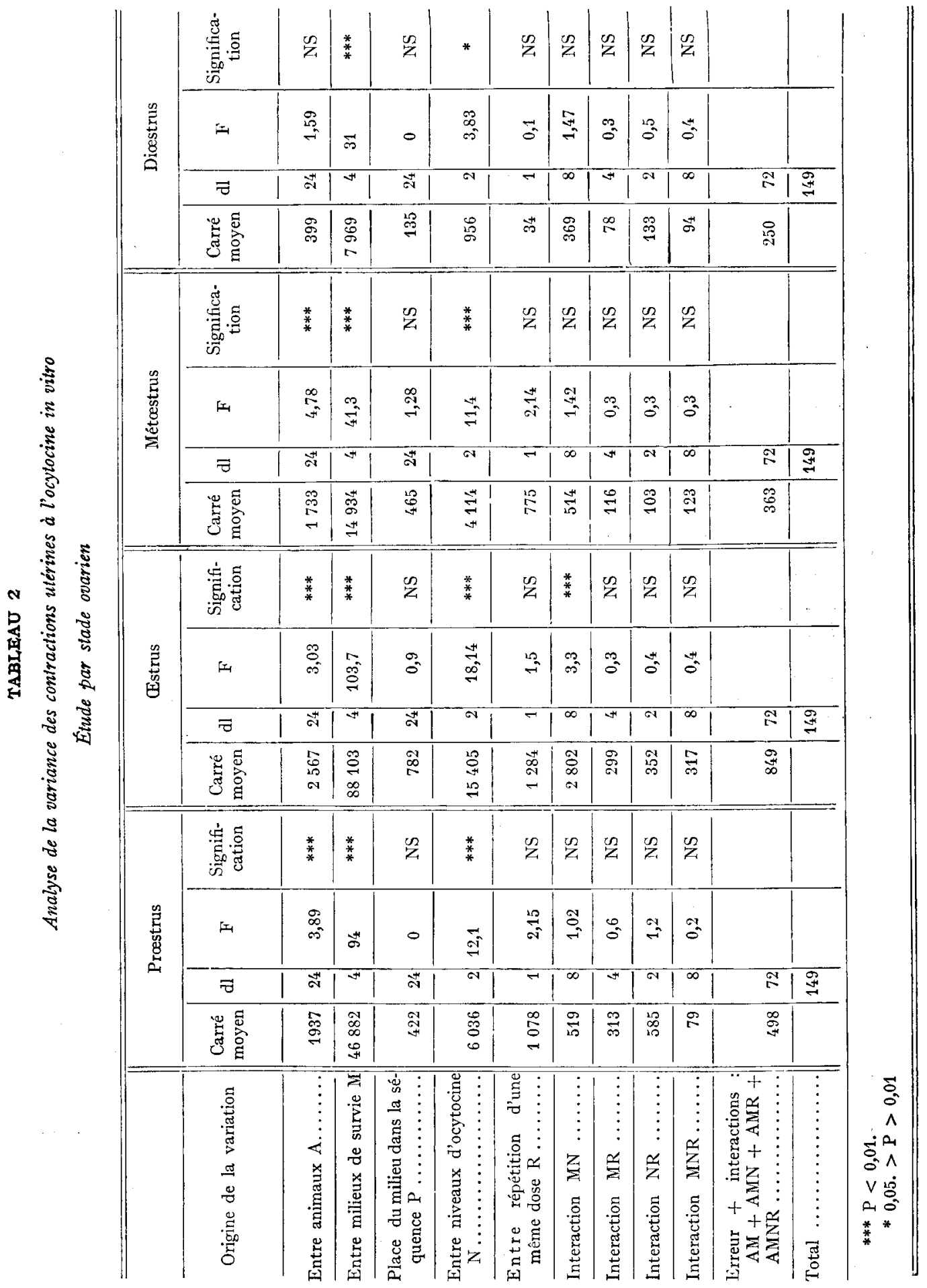


SENSIBILITÉ DE L'UTÉRUS DE RATTE A L'OCY'TOCINE

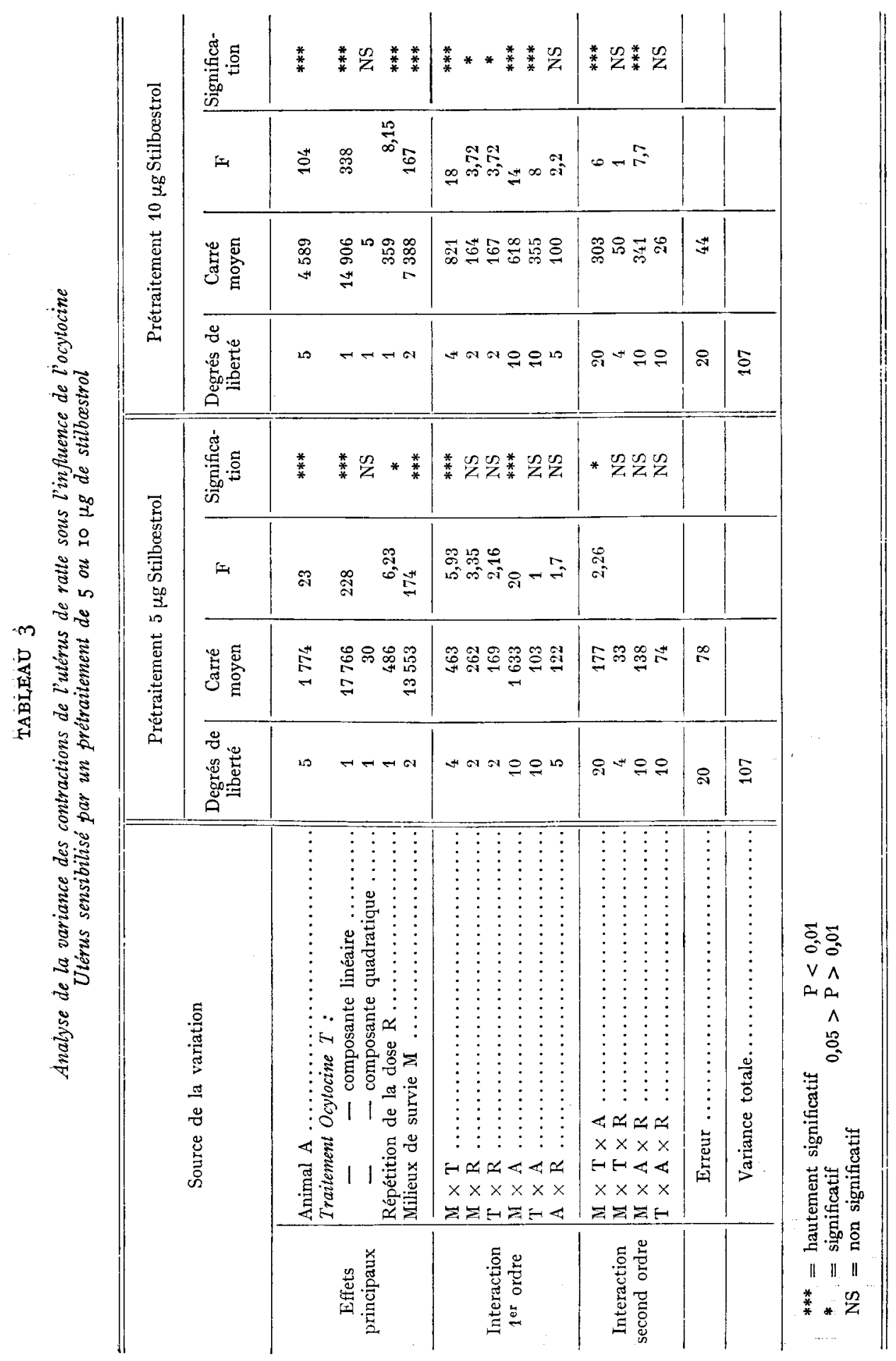


II. - Analyse des réponses des utérus ayant subi un prétraitement par $5 \mu \mathrm{g}$ ou Io $\mu \mathrm{g}$ de stilbostrol

Le tableau 3 confirme dans le cas d'un prétraitement les conclusions générales tirées du paragraphe précédent.

Toutefois, certaines interactions significatives, en particulier les interactions de premier ordre où figure le facteur milieu de survie sembleraient montrer que les variances dues aux effets : traitement, animal, ou répétition diffèrent selon le milieu considéré.

La comparaison globale des prétraitements se révèle faiblement significative $(0, \mathrm{I}<\mathrm{P}<0,05)$. Nous verrons plus loin que cette faible différence moyenne s'explique par le fait que selon le milieu en cause, l'ordre des deux prétraitements est inversé

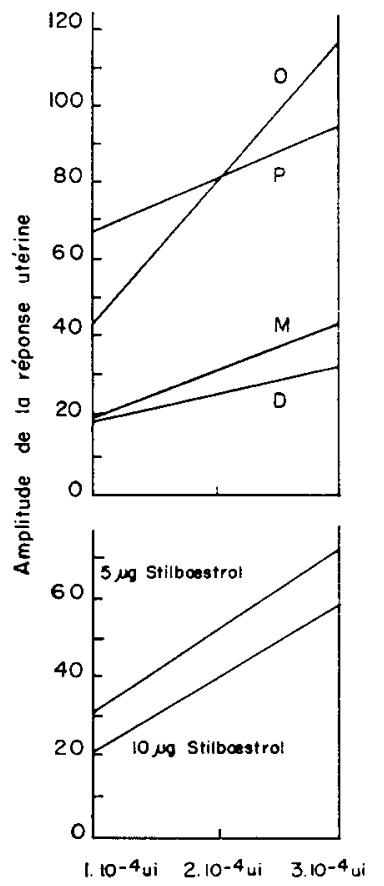

Pharmacopee

Britannique 1953

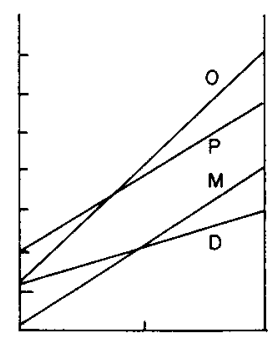

$\begin{array}{ll}P=\text { prostrus } & M=\text { metcestrus } \\ O=\text { costrus } & D=\text { dicestrus }\end{array}$

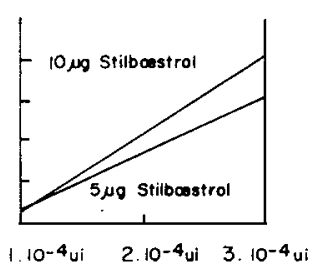

Beouvillain
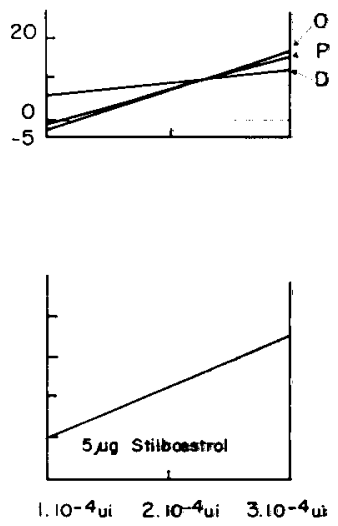

Jalon Holton

GRAPHIQUE I. - Représentation graphique des végressions de la réponse utérine en fonction de la dose d'ocytocine

III. - Caractéristiques de la régression de la réponse de l'utérus de ratte selon le stade ovarien et le milieu de survie (Graphique I)

Nous avons rassemblé sur le tableau 4 les équations des droites de régression pour les solutions et les stades où ces régressions sont significatives.

En ce qui concerne l'influence du stade ovarien au moment où l'utérus est prélevé, nous voyons que les meilleures régressions se trouvent en œstrus d'abord, puis en prœstrus. La pente semble toujours plus forte en œstrus qu'en prœstrus.

La comparaison des régressions deux à deux (tabl. 6) nous montre que :

- la solution de la Pharmacopée britannique est la meilleure pour 1'amplitude 


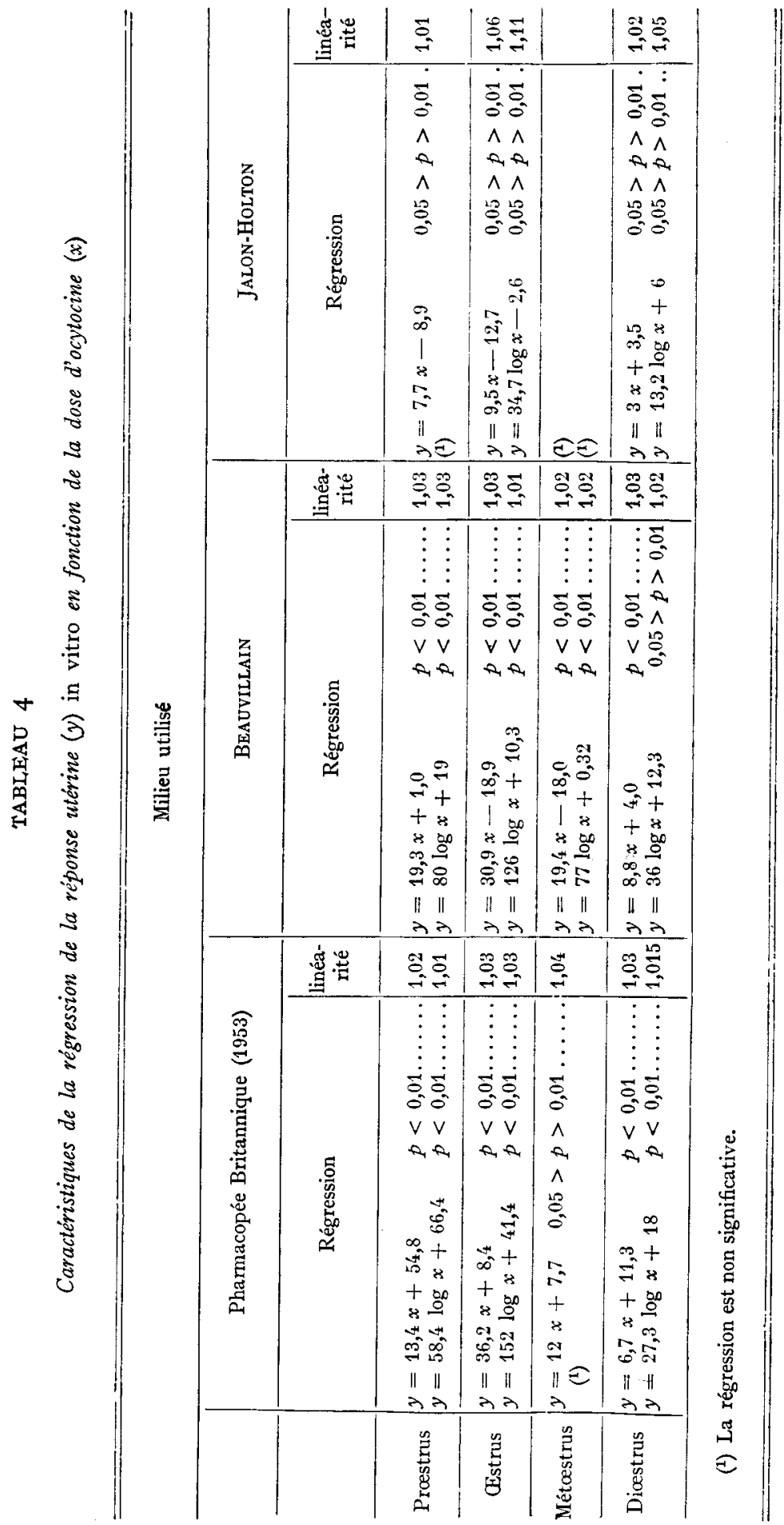


des contractions qu'elle permet. Les pentes sont significativement différentes entre leprœstrus et l'œstrus (œstrus > prœstrus), les moyennes de réponses sont identiques.

- dans la solution de Beauvilitain qui vient en second rang les régressions en œstrus et en prœstrus sont identiques. Les différences entre BEAUVILLAIN œestrus ou prœstrus et Pharmacopée britannique, œstrus ou procstrus sont toutes hautement significatives;

- dans la solution de JALON-Hol,Ton, les pentes sont faibles à la limite de la signification ; l'amplitude des réponses réduite.

Nous n'avons jamais pu obtenir de régression significative pour les solutions. utilisées habituellement avec la technique de perfusion (GINSBURG et SmrTh, FitzPATRICK).

Nous remarquons enfin que l'ajustement de la régression de la réponse, selon la valeur absolue de la dose d'ocytocine ou selon le logarithme de cette dose est linéaire pour les deux possibilités, sauf en ce qui concerne le milieu de JALON-HOLTON. Ceci peut s'expliquer par le fait que la gamme des doses d'ocytocine choisies pour cet essai est très étroite, ce qui ne favorise pas la mise en évidence d'une régression. habituellement admise comme logarithmique.

\section{IV. - Caractéristiques de la régression de la réponse de l'utérus de ratte} ayant subi un prétraitement de $5 \mu \mathrm{g}$ ou ro $\mu g$ de stilbostrol (tabl. 5 et graphique I)

Nous mettrons à part la régression pour le milieu de JALON-HoLTON pour lequel. le prétraitement modifie peu l'allure de la droite obtenue en prœstrus ou en cestrus.

Pour les deux autres solutions, les résultats semblent opposés :

- avec la solution de la Pharmacopée britannique le prétraitement à $5 \mu \mathrm{g}$ de stilbœstrol donne une régression dose-réponse supérieure à celle obtenue avec Io $\mu \mathrm{g}$ (hautement significative, tab1. 6) ;

- le résultat est inversé avec la solution de BEAUVIL, AIN mais cette différence n'est pas statistiquement significative (tabl. 6).

\section{TABLEAU 5}

Caractéristiques de la régression de la réponse à l'ocylocine de l'utérus de ratte ayant subi un prétraitement de $5 \mu \mathrm{g}$ ou тo $\mu \mathrm{g}$ de stilboestrol

\begin{tabular}{|c|c|c|c|c|c|}
\hline Milieu & $\begin{array}{l}\text { Prétraite- } \\
\text { ment }\end{array}$ & $\begin{array}{l}\text { Ajustement } \\
\text { arithmétique }\end{array}$ & Linéarité & $\begin{array}{c}\text { Ajustement } \\
\text { logarithmique }\end{array}$ & Linéarité \\
\hline $\begin{array}{c}\text { Pharmacopée } \\
\text { Britannique } \\
(1953)\end{array}$ & $\begin{array}{r}5 \mu g \\
10 \mu g\end{array}$ & $\begin{array}{l}y=21 x+10 \\
y=19,6 x+0,6\end{array}$ & $\begin{array}{l}1,003 \\
0,971\end{array}$ & $\begin{array}{l}y=90,9 \log x+30 \\
y=80,1 \log x+19\end{array}$ & $\begin{array}{l}0,969 \\
0,985\end{array}$ \\
\hline BEAUVILLAIN & $\begin{array}{r}5 \mu g \\
10 \mu g\end{array}$ & $\begin{array}{l}y=12,8 x-8,2 \\
y=18,6 x-14,3\end{array}$ & $\begin{array}{l}0,996 \\
0,973\end{array}$ & $\begin{array}{l}y=50,1 \log x+4,3 \\
y=77,3 \log x+2,9\end{array}$ & $\begin{array}{l}1,045 \\
0,971\end{array}$ \\
\hline JALON HOLTON & $\begin{array}{r}5 \mu \mathrm{g} \\
10 \mu \mathrm{g}\end{array}$ & $\begin{array}{l}y=12,7 x-2,7 \\
\text { Régression non sign }\end{array}$ & $\begin{array}{l}0,997 \\
\text { cative }\end{array}$ & $y=52,9 \log x+8,9$ & 0,972 \\
\hline
\end{tabular}




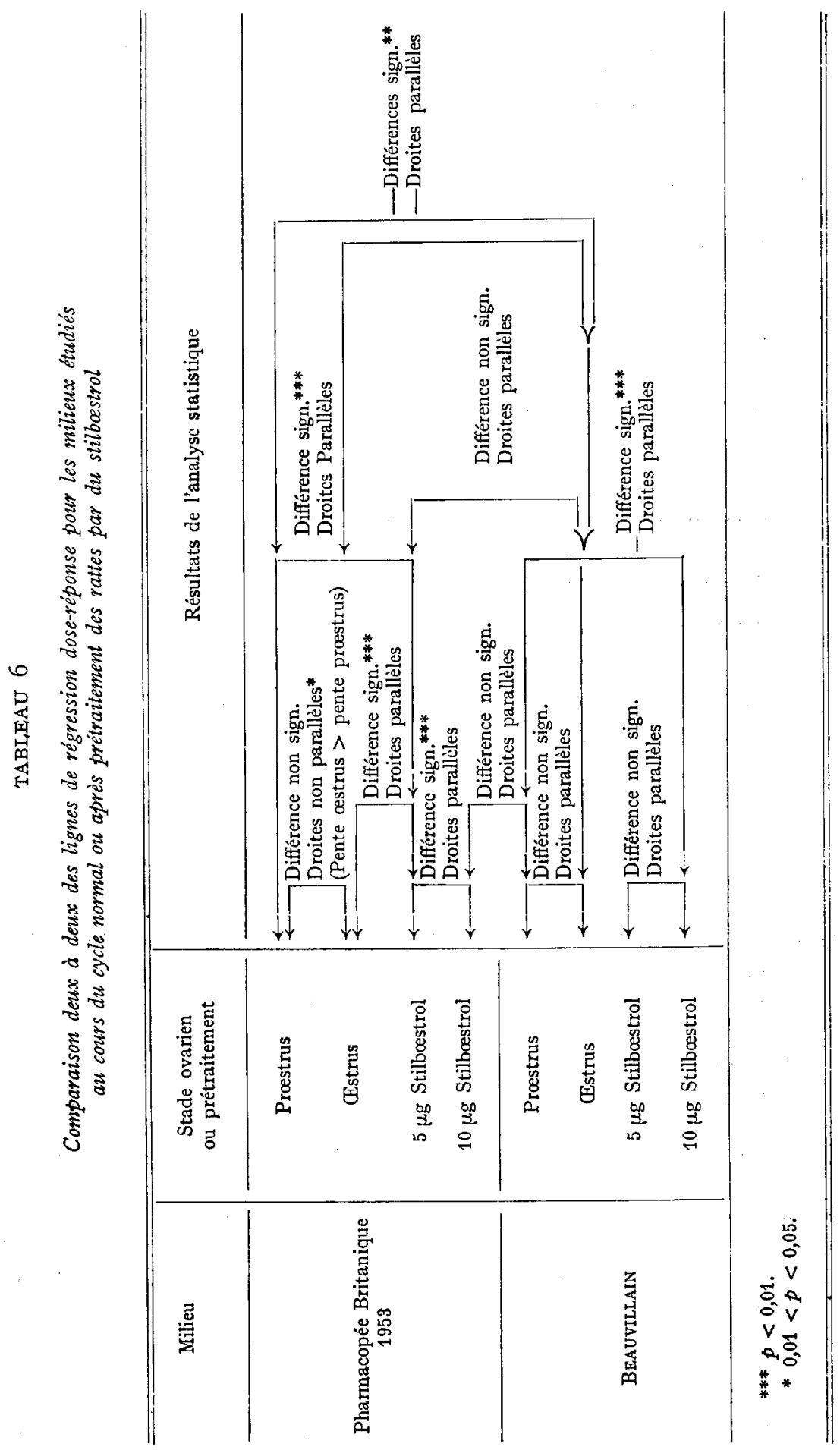


V. - Comparaison générale des droites de régression.

Dose-réponse de l'utérus maintenu en survie dans différents milieux et en fonction de son état physiologique

Le tableau 6 donne les résultats de l'analyse statistique des comparaisons des droites de régression prises deux à deux. Nous nous sommes limités en ne présentant que celles qui permettent des conclusions intéressantes.

Pour chacun des deux milieux : Beauviliarn et Pharmacopée britannique, le choix des utérus en œstrus ou en prœstrus permet toujours une régression signifitivement supérieure à celle obtenue avec le prétraitement $5 \mu \mathrm{g}$ ou Io $\mu g$ de stilbœstrol.

L'ensemble des comparaisons effectuées permet de classer les combinaisons : milieux, stade ovarien et prétraitement, dans l'ordre décroissant suivant :

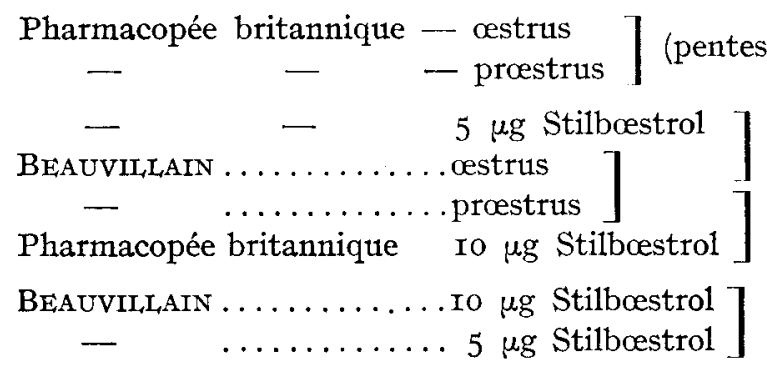

Les accolades indiquent les couples de combinaisons dont les régressions sont non significativement différentes $(\mathrm{P}>0,05)$.

\section{DISCUSSION ET CONCLUSION}

En ce qui concerne le problème du choix de l'utérus en fonction du stade physiologique, nos résultats sont en accord avec les auteurs qui préconisent le dosage de l'ocytocine sur un organe prélevé en prœstrus ou en ostrus. Le métœstrus et le diœstrus étant à rejeter si l'on désire obtenir une préparation très sensible.

La sélection des animaux par frottis vaginal est donc indispensable à ce choix. C'est une opération rentable, car elle conditionne la qualité du dosage.

Le prétraitement des femelles par une injection d'œstrogène (Stilbœstrol, $5 \mu \mathrm{g}$ ou Io $\mu \mathrm{g}$ par $200 \mathrm{~g}$ ) I7 à I 8 heures avant l'abattage est efficace car, dans ce délai, il déclenche chez ces animaux un début de cycle et le dosage se fait alors en prœstrus ou en début d'œstrus. La sensibilité obtenue par ce moyen est toujours supérieure à celle observée en métœstrus ou en diœstrus, mais cependant n'égale pas celle constatée durant le prœstrus ou l'œstrus normaux. Cette technique, aux doses où nous avons utilisé les ostrogènes, peut donc être considérée comme inférieure à celle du choix par frottis vaginal, mais toujours supérieure au tirage des animaux au hasard dans une population quelconque.

Notre attention a été particulièrement attirée par l'importance de la variance " animal ". Si une certaine homogénéité des résultats a été observée en ce qui concerne 
le choix du stade dans le cycle ovarien, il n'en reste pas moins que pour un stade physiologique donné, la variance entre les individus reste grande. Sans vouloir apporter de solution définitive à cette importante source de variation, nous pensons qu'elle peut être réduite par une sélection convenablement menée sur deux souches séparées dont on utiliserait les croisements de première génération pour le dosage. Cette technique aurait pour résultats de diminuer et de stabiliser la variance animal, et d'augmenter la sensibilité de la préparation utilisée en $F_{1}$.

Il reste enfin le problème du choix du milieu de survie à utiliser, il n'y a pas dans ce domaine d'option définitive à prendre, car les combinaisons des sels qui les composent sont en très grand nombre. Les interactions révélées par 1'analyse statistique semblent même montrer que les différents effets ne sont pas indépendants ; de ce fait, le choix du milieu de survie peut dépendre du but que l'on se propose :

- dosage pharmacologique d'ocytocine dans des extraits riches,

- dosage d'ocytocine dans des extraits très pauvres et peu abondants,

- comparaison de substances ocytociques (Munsick, Ig60).

La comparaison des résultats avec l'examen détaillé des compositions permet d'avancer quelques remarques sur l'influence des concentrations en divers ions, sur la qualité des réponses utérines, l'expérience in vitro étant poursuivie en cuve et les doses d'ocytocine appliquées étant de 1'ordre de o,I mU par $\mathrm{ml}$.

La comparaison des trois solutions différentes dans leur concentration en magnésium et par ailleurs identiques nous montre que :

- l'absence de magnésium chez le Rat, comme chez le Cobaye (VAN Drke, et Hasting, I928), réduit considérablement la sensibilité de l'utérus aux faibles doses d'ocytocine,

- la solution de la Pharmacopée britannique avec 0,026 $\mathrm{mM}$ se révèle supérieure à la solution de BEAUVILLAIN $(0,052 \mathrm{mM} / 1)$ l'optimum se situerait donc entre o et $0,052 \mathrm{mM} / 1$.

La présence de calcium à un taux optimum ne semble pas suffisante pour assurer une sensibilité convenable à l'ocy tocıne d'un utérus choisi en prœstrus ou en 'œstrus La solution de JALON-HolTon ayant le même taux de calcium que les solutions de 1a Pharmacopée britannique ou de BEAUVILLAIN, mais sans magnésium se révèle très inférieure à ces dernières.

L'excès de calcium joint à l'excès de magnésium (solution de Fitzpatrick) ne permet pas l'utilisation des contractions d'un utérus en cuve, pour le dosage de l'ocytocine.

Enfin, la déficience en sodium (solution de Ginsburg et Smith), malgré la présence de calcium et de magnésium en proportions convenables provoque d'abondantes contractions spontanées sans que nous ayons pu parvenir à la période de stabilité décrite par Marshall (I963).

Cette expérience confirme le fait que l'équilibre ionique que LOEWE (I926) décrivait entre sodium, potassium et calcium est à étendre et à rechercher entre les autres ions tels que magnésium, équilibre qui permet ou ne permet pas l'action des ions $\mathrm{Ca}^{++}$même lorsque ceux-ci sont présents en quantité satisfaisante.

En conclusion, il ressort de cette expérience qu'en cuve classique, le dosage de l'ocytocine dans des extraits pauvres en hormones doit être fait sur des utérus de Rats pesant I30 à I9o g. Ces animaux seront abattus en prœstrus ou en oestrus 
déterminés par frottis vaginal. Dans l'état actuel de nos connaissances, nous pensons que l'organe doit être maintenu en état de survie convenable par immersion dans la solution de la Pharmacopée britannique (I953) ou dans une solution qui serait de composition très voisine, maintenue à $32^{\circ}$ et dans laquelle barbotte un mélange d'oxygène et de gaz carbonique (95 p. Ioo et $5 \mathrm{p}$. IOo).

Reçu pour publication en mai I 964 .

\section{SUMMARY}

\section{STATISTICAL ANALYSIS OF THE SENSITIVITY OF THE RAT UTERUS in vitro TO OXYTOCIN}

The sensitivity of the rat uterus to oxytocin was tested in relation to the stage of the ovarian cycle in the course of which the uterus was removed.

Five survival solutions were used :

$$
\begin{aligned}
& \text { British Pharmacopoeia, 1953; } \\
& \text { Jalon solution (HoLton, I948); } \\
& \text { Beauvillain solution (1943); } \\
& \text { Ginsburg and Smith solution (1961). }
\end{aligned}
$$

The doses of oxytocin applied to the uterus were

$$
\text { I. } 10^{-4}, 2.10^{-4}, 3.10^{-4} \mathrm{IU} \text {. }
$$

In the course of a second experiment, the uteri were sensitised to oxytocin by an injection of 5 to $10 \mu \mathrm{g}$ of stilbœstrol per $200 \mathrm{~g}$ of live weight, I7 to 18 hours before the removal of the uterus.

Table 2 gives the results of the analysis of variance for the responses at different stages of the ovarian cycle.

Table 3 gives the results of the analysis of variance for the responses of uteri sensitized by cestrogens.

The equations for the linear dose-response or $\log$ dose-response regressions are given in tables 4 and 5 , and are represented graphically in fig. $\mathrm{I}$.

The comparisons carried out allow the combinations to be classed thus : solution, ovarian stage, and pre-treatment, in the following order of decreasing quality :

$\begin{array}{ll}\text { British Pharmacopoeia : ostrus. } \\ \text { British Pharmacopoeia : prostrus. } \\ \text { British Pharmacopoeia : } 5 \mu \mathrm{g} . \\ \text { Beauvillain } & : \text { ostrus. } \\ \text { Beauvillain } & : \text { prostrus. } \\ \text { British Pharmacopoeia }: \text { ro } \mu \mathrm{g} . \\ \text { Beauvillain } & : \text { ro } \mu \mathrm{g} . \\ \text { Beauvillain } & : 5 \mu \mathrm{g} .\end{array}$

The Jalon solution, described by HoLTon (1948), permits only very weak responses by the uterus to the doses of oxytocin used.

The solutions of GinsBURG and SMitri and of FitzPatrick, which those authors used in the superfusion technique, do not give a significant dose-response regression in the method using a standard bath.

\section{RÉFÉRENCES BIBLIOGRAPHIQUES}

Beauvillain A., I943. Titrage des préparations posthypophysaires par leur seuil d'action sur l'utérus isolé de Rat adulte. C. R. Soc. Biol. (Paris), 137, 284-285.

Berger E., Marshall J. M., I96r. Interactions of oxytocin, potassium and calcium in the rat uterus. Amer. J. Physiol., 201, 931-934.

BonNey W. R., FERGUSON J. K. W., 1950. Reactions of isolated uterine muscle of rats and guinea pigs. Arch. Int. Pharmacodyn., 83, 566-572. 
British Pinnrmacopoeia, i953. General Medical Council edts, Pharmaceutical Press, London, 822.

Brouna L., Simonnet H., I926. Influence de l'injection préalable de liquides folliculaire sur la réponse de l'utérus à l'hypophyse. C. $R$. Soc. Biol. (Paris), 95, 674-676.

Brovha L., SimonNeT H., 1927. Recherches expérimentales sur la régulation hormonale de la contractilité utérine. Arch. int. Physiol., 29, 95-120.

BuRN J. H., Dale H. H., 1922. On the physiological standardization of extracts of the posterior lobe of the pituitary body. Annual Rep. Med. Res. Council, London, ${ }^{\circ} 69$ Special Report, 5-52.

Clark A. J., Knaus H. I., Parkes A.S., 1925. CEstrous variations of uterine activity in the rat. J. Pharmacol., 26, 359-369.

Csapo I. A., 1956. Relation of threshold to the K gradient in the myometrium. J. Physiol., 133, 145-1 58.

Csapo I. A., Kurivama H. A., I963. Effects of ions and drugs on cell membrane activity and tension. in the postpartum rat myometrim. J. Physiol., 165, 575-592.

Dale H. H., Laidlaw P. P., I912. A method of standardizing pituitary (infundibular) extracts. J. Pharmacol., 4, 75-95.

Duffner G. J., Whitehead R. W., ig4r. The effects of oestrone, diethylstilbostrol and testosterone on the in vitro response of the spayed rat uterus to Pitocin. J. Pharmacol., 73, 296-303.

Durrant E. P., Rosenfeid S., 193r. Activity of the isolated uterus and its relation to the cestrous cycle in the albino rat. Amer. J. Physiol., 98, $153^{-1} 55$.

FinNey D. J., 1952. Statistical method in biological assay, Griffin C. and Co. Ltd, edts, London.

Fitzpatrick R. J, I96r. The estimation of small amounts of oxytocin in blood, in Oxylocin Caldeyro Barcia R., and Heller H. edts, Pergamon Press, Oxford, 358-377.

Flatters M., 1954. Uber die Vorzüge der Verwendung von Ratten-Literi im Proöstrus zur Auswertung von Oxytocin. Arch. exper. Path. t. Pharmakol., 221, 171-176.

Gaddum J. H., 1953. The technique of superfusion. Brit. J. Pharmacol., 8, 321-326.

Garcia de Jalon P., Bayo Bayo J., Garcia de Jalon M., 1945. Sensible y nuevo metodo de valoracion de adrenalina en utero aislado de rata. Farmacoter. act, n $^{\circ}$ I I, 31 $3-3 \mathrm{I} 8$.

Genell S., I938. Die Bedeutung des Magnesiumions für die Motorik des überlebenden Lterus. Skand. Arch. Physiol., 80, ז 24-1 32.

Genell S., 1940. Studies on the muscular physiology of the genital tract. Acta Physiol. Scand., 1, r 39-148

Ginsburg M., Smiti M. W., 1959. The fate of oxytocin in male and female rats. Brit. J. Phar macol. 14, $3^{27-333 .}$

Goto M., CSAPO I. A., I959. The effect of ovarian steroides on the membrane potential of uterine muscle. J. Gen. Physiol., 43, 455-466.

GUerne J. M., Stutınsky F., I96r. Études in vivo des variations de la sensibilité à_l'ocytocine de l'utérus de Ratte cyclique. J. Physiol. (Paris), 53, 357-358.

Guggenheim M., r9i4. Beitrag zur Kenntnis der Wirksamen Prinzips der Hypophyse. Biochem. Z., 65, $189-218$.

HARNE O. G., 1932. A study of the excised uterus of the rat, its volume displacement and irritability to pituitrin with reference to the cestrous cycle. Amer. J. Physiol., 100, 33-1-338.

Holton P., 1948. A modification of the method of Dale and LaIdlaw for standardization of posterior pituitary extracts. Brit. J. Pharmacol., 3, 328-334.

Jornston F., 1958. Hethods used for the assay of extracts which contain the hormones of the posterior pituitary gland. Lab. practice, $7,575-577$.

JUNG H., I $96 \mathrm{I}$. The effect of oxytocin on mechanism of uterine excitation, in Oxytocin Caldeyro Barcia R. and Heller H. edts, Pergamon Press, Oxford, 87-99.

KNaUS H. II., Clark A. J., 1925. The action of certain drugs and ions on the rat's uterus. J. Pharmacol., 26, $347^{-} 35^{8}$.

Kumagai H., Ebashi S., Takeda F., I952. A newer method of biological standardization of the oxytocic principle of posterior pituitary. Jap. J. Pharmacol., 2, 65-81.

KURIYAMA H., CSAPO I. A., 1959. The "evolution " of membrane and myoplasmic activity of uterine muscle. Biol. Bull., 117, 417-418.

Locke F. S., I 896. Artifical fluids as uninjurious as possible to animal tissue. J. Boston Soc. Med. Sci., $1,2-3$.

LOEWE S., I 926 . Ionen dyskasie, ein grafisches Hilfmittel zum Studium der Folzen von Ionen Zusammensetzung biologischa Salzlösungen. Biol. Z., 167, 92-99.

Long J. A., Evans H. M., I922. The œstrus cycle in the rate and its associated phenomena. Mem. Univ. Calif., 6, I-I42.

Marshalt J. M., I959. Effect of estrogen and progresterone on single uterine muscle fiber in the rat. Amer. J. Physiol., 197, 935-942.

Marshall J. M., 1962. Regulation of activity in uterine smooth muscle. Physiol. Rev., 42, suppl. 5, $213-227$.

Marshall J. M., I 963 . Behavior of uterine muscle in Na-deficient solutions; effects of oxytocin. Amer. J. Physiol., 204, 732-738. 
Munsick R. A., I960. Effect of magnesium ion on the response of the rat uterus to neurohypophysial hormones and analogues. Endocrinology, 66, 451-457.

Robson J. M., Sharaf A. A., I95I. The action of sodium ostrone sulfate on the uterus of the mouse in vitro. J. Endocrin., 7, 221 .

SchneIder W., StumpF C., I953. Uber den Einfluss des Sexualcyclus auf Hypophysenhinterlappenextrakt auswertungen nach der Methode von P. Holton. Arch. Int. Pharmacodyn., 94, 406-415.

SiaUlis B., Sollman T., I927. The response of the circular and longitudinal muscle of Rabbits ileum to physical and mechanical conditions. Amer. J. Physiol., 81, 579-605.

Tyrode M. V., igro. The mode of action of some purgative salts. Arch. Int. Pharmacodyn., 20, $205^{-223}$.

VAN Dyke H. B., Hastings A. B., 1928. The response of smooth muscle in different ionic environments. Amer. J. Physiol., 83, 563-577.

VAN Dyke I1. B., I96I. Some features of the pharmacology of oxytocin, in Oxytocin, Caldeyro Barcia R. and Heller II. edts, Pergamon Press, Oxford, 48-66.

Walker J. M., 196r. In Hormones in Blood, Acad. Press N. Y. London, 149-163. 